“ (C) 2017 IEEE. Personal use of this material is permitted. Permission from IEEE must be obtained for all other uses, in any current or future media, including

reprinting/republishing this material for advertising or promotional purposes, creating new collective works, for resale or redistribution to servers or lists, or reuse of any copyrighted component of this work in other works." 


\section{Mobility Management of D2D Communication for the 5G Cellular Network System: A Study and Result}

\author{
Shouman Barua \\ University of Technology, Sydney \\ Australia \\ Email: Shouman.Barua@student.uts.edu.au
}

\author{
Robin Braun \\ University of Technology, Sydney \\ Australia \\ Email: Robin.Braun@uts.edu.au
}

\begin{abstract}
Wireless devices connected to the networks have been increasing remarkably for the last couple of decades. Ubiquitous voice and data connection are the key requisite for the next generation of wireless technology. Device to Device communication is widely known as D2D communication which is a new paradigm of cellular communication, and it was initially proposed to boost the network performance. It takes place when two devices communicate directly without taking significant help from the base station. Similar to other wireless communication systems, mobility management for the D2D communication is a big challenge which is yet to explore properly. Mobility management of $D 2 D$ communications was enlightened in few papers. It should be managed in such a smart way in a cellular network that should enable lower latency, lower power consumptions, less complexity and last but not the least possible uninterrupted data connections which are primarily the requirements of next generations mobile cellular network. This paper extends our algorithm paradigm of mobility management of D2D communication that was proposed and published previously. We come up with the simulation results in this paper that validate our proposed model. However, we also highlight the D2D communications and mobility issues within it.
\end{abstract}

Index Terms-Device to Device Communication, Mobility Management, 5G, Inband D2D, Underlay D2D.

\section{INTRODUCTION}

Mobile communication is fundamentally regarded as a recent innovation, and most of its features were introduced after the 1980s. The era of cellular communication has been divided into several generations. Next generation mobile system which is also known as Fifth Generation (5G) is expected to fulfill the major demands of data services in telecommunication system along with new era of Internet of Things (IoT). 5G mobile network refers to the next major phase of mobile telecommunications system beyond the currently available IMT-Advanced/4G standards. 5G systems have not been standardized yet, and it is still an open question whether it fits all technologies to address all use cases and requirements or not. It is assumed not only about to boost the data rate but also the extensive connections in addition to the data rate in $4 \mathrm{G}$.

Existing 4G architecture needs to be changed considerably to get the $1000 \mathrm{x}$ data rate for the $5 \mathrm{G}$ communication system.
Mobile cellular communication has been designed historically based on cells as a primary unit for the radio access network to ensure the coverage and capacity. Network densification is a significant trend of any cellular generation. As heterogeneous network was a significant modification in the $4 \mathrm{G}$ network, the $5 \mathrm{G}$ network is assumed to be changed into device-centric architecture [1]. It will modify the network densification in different directions. Apparently, no-edge network, i.e., a uniform network solution is a trend and challenge for the 5G cellular network. Moreover, using the smarter devices can effectively change the concept of cell architecture since D2D or M2M (Machine to Machine) are going to be a major factor day by day. When it comes to an issue of communication between two end users, smooth mobility management plays an important role to keep the quality up to the threshold. Communication between two ends can effectively be realizable if a smart movement system could be integrated. Mobility management for the D2D communication is an early stage now. We need to have a proper mobility management algorithm for the D2D communication so that it could be implemented in the 5G cellular network system. The objective of this paper is to prove our previously proposed algorithm regarding the mobility management of D2D communication with simulation results that was the next task in our previous paper [2].

The rest of the paper is structured such as next section is for the basics of D2D communications system along with the problem statement of mobility management. Section three highlights the novel approach of mobility management for the D2D communication system that was initially proposed in our paper [2]. Section four is dedicated to the simulation parameters and results. Finally, section five concludes the paper with focusing the major works done and future directions.

\section{D2D COMMUNICATION AND THE PROBLEM OF MOBILITY MANAGEMENT}

D2D Communications is another direction that a wireless communication system can take, where a device directly communicates with another device to increase the overall 
throughput and spectrum efficiency. This offloads base station traffic. It is a communication technology that facilitates any device to communicate directly without the support of major infrastructure, i.e. a base station, during the data transfer period. Base stations only control the devices to enable the communication between them.

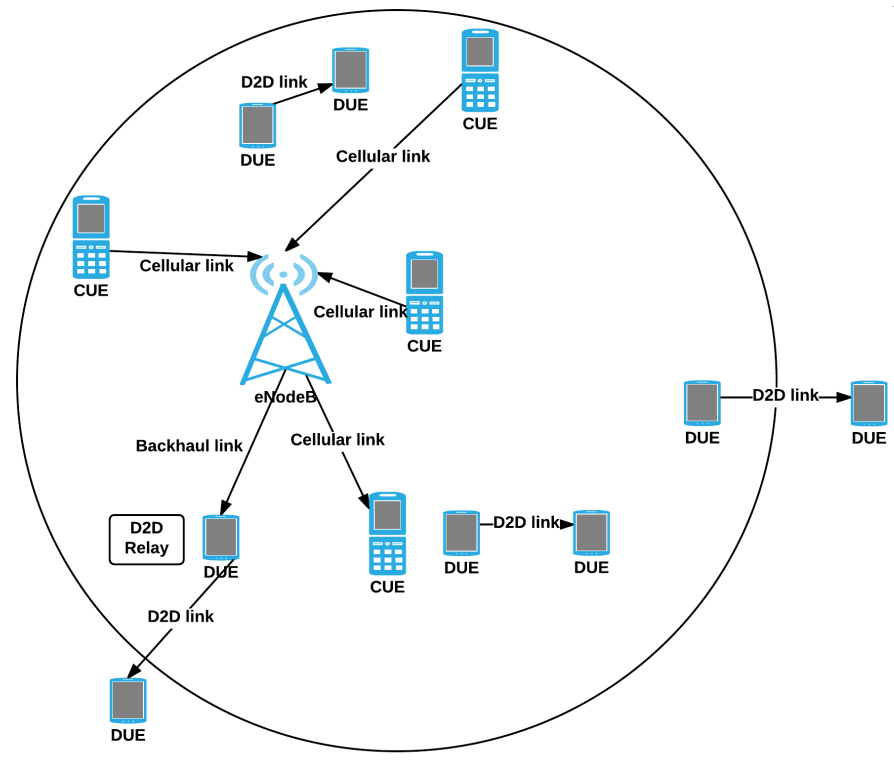

Fig. 1. D2D communication in a cellular system.

There are several ways where D2D communication could be used and improves the overall performance. If a device is located out of the coverage area of a base station, Signal to Interference plus Noise Ratio (SINR) is usually low, and the device might not be able to communicate with others using base station. However, if the device is located within a proximity area of another device, then overall coverage area might be extended by using the second device as a relay. In that case, overall spectrum utilization increases. Fig.1 illustrates an overall view of D2D communication in a cellular network. Paper [3] mentioned some other applications of D2D communications like a Vehicle to Vehicle (V2V) communications, cellular offloading, content distribution, gaming, social networking, etc. On the other hand, a network with the D2D communication enabled could significantly increase the overall energy efficiency. As a device transmits and receives from the very short distance, they do not require massive power to transfer to the another device and base station do not need to communicate with maximum power while the device is used as a relay.

D2D communication still requires an efficient protocol and algorithm to handle these communication scenarios. Key challenges of D2D communication are to find the real opportunities to establish the connection, duplexing structure of uplink/downlink communication, designing devices that are
D2D enabled from both protocol and hardware perspective, accounting the extra overheads to control the communication and estimating the channel [1]. Location of the D2D users could be calculated using the direction of arrival estimation techniques [4] [5]. A discussion of full feature and opportunity of D2D communication is out of the scope of this paper. Survey paper [3] discusses all the basics of D2D communications. However, Fig. 2 mentions of the major classifications of D2D. A brief explanation of each classification is also out of the scope of this paper.

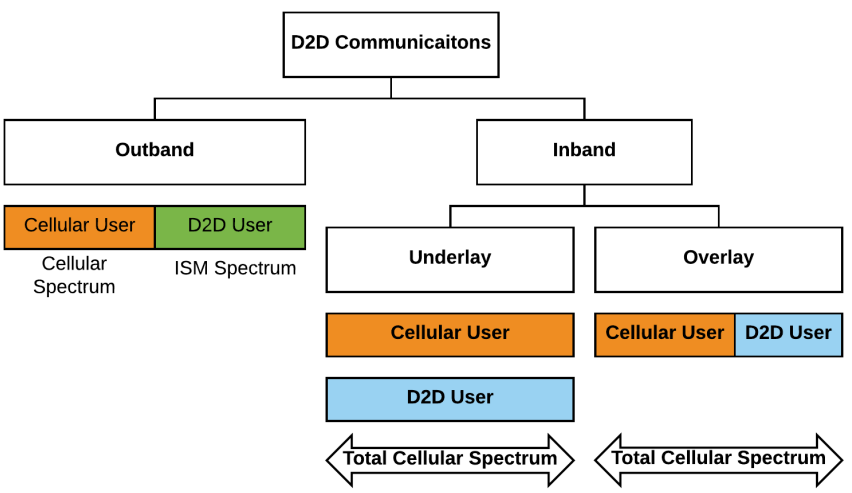

Fig. 2. Major classification of D2D communications

Mobility management for the LTE-A is so far well standardized and already implemented. A role of mobility model considering the energy and bandwidth efficiency is proposed in [6] for the LTE-A network. Paper [?], [7], [8] provides a technical overview of handover and mobility management for the heterogeneous network (4G) in LTE-A. Authors in this paper investigate A3 events that take control of the entire handover parameters. Usually, the mobility of a user is mentioned broadly in three different states, i.e., idle, active, and detached mode. Handover takes place when the UE moves from one location to another location that requires the intervention of new base station to provide the user a smooth service. Detailed handover process of a UE for the LTE-A network is also out of the scope of this chapter. This paper describes the entire handover process for only the D2D communication systems.

However, the standard handover does not apply to the D2D communication process as this communication involves and must satisfy additional parameter issues for the both devices. So far, few papers were published based on the mobility management of D2D communications. Reducing latency is the primary target for any communication. Paper [9] considered two different kinds of solution that could reduce the latency considerably. Authors justified the solutions with some simulation results. These two categorized smart solutions are D2Daware handover solution and D2D-triggered handover solution. Authors propose the control of devices by the single BS. That is because when the difference eNodeBs control them, 
latency is increased substantially as a result of extra overhead to communicate with two different base stations. It causes performance degradation. In our paper, we propose D2Daware handover provided the condition is that source eNodeB will postpone the handover until controls of both devices are satisfied by target eNodeB2. In the case of D2D-triggered handover solution, authors mention a signaling flow chart that shows a new D2D user takes the control to join a group communication. Authors aim to cluster the total members of a D2D group within a minimum number of eNodeBs or cells so that total signaling overheads resulted by the interBS communication are reduced. Paper [10] also propose a handover mechanism for D2D communication. However, our proposal also considered some factors in addition to this paper like spectrum efficiency based on the total sum-rate using only uplink resources, mode selection and more realistic model of path loss.

\section{PROPOSED MODEL}

Referring to our previously published paper [2] where we proposed a new model and left the simulation result as a future work, we extend the model further along with the simulation results in this paper. We consider the same parameters and mathematical models mentioned in paper [2]. A glimpse of this mobility model is mentioned in Fig. 3 where D2D transmitter (DUETx) is moving towards another eNB. We consider only the Time Division Duplex (TDD) configuration for our proposed model. In that case, all the standard mechanism that are usually applied for the TDD are also applicable to our proposed model accordingly.

In this model, we also consider the underlay inband D2D communication to make sure that spectral efficiency is maximized. In that case, same resources are used by both $\mathrm{D} 2 \mathrm{D}$ and cellular users. We assume that D2D users use same uplink resources as the cellular users. So, every D2D pair is associated with one cellular device where they share the same uplink resources for the communications. Some frequency reuse algorithms are discussed in paper [11]-[14] for the downlink. But we consider only uplink algorithms for our model to increase the spectrum utilization. Our approach is to increase the total sum-rate where total sum-rate includes the sum-rate of D2D pairs $r_{\mathrm{D} 2 \mathrm{D}}$ and sum-rate of cellular users $r_{\text {Cellular }}$ as follows:

$$
R_{\text {Sum }}=r_{\mathrm{D} 2 \mathrm{D}}+r_{\text {Cellular }}
$$

We consider the group handover to the target eNB as already mentioned in paper [9] and discussed in the previous section. It reduces the latency and other functional overheads. Our proposed model also consider the paper [15] where a normal D2D operation is offered without the scope of handover possibilities. In our previous paper [2], we proposed mobility management in this model in addition to the general D2D operational model. Fig. 3 shows detailed scenarios where channel link, gains, a position of the eNB, D2D users, cellular users and mobility directions are mentioned explicitly along with the mathematical model [2].

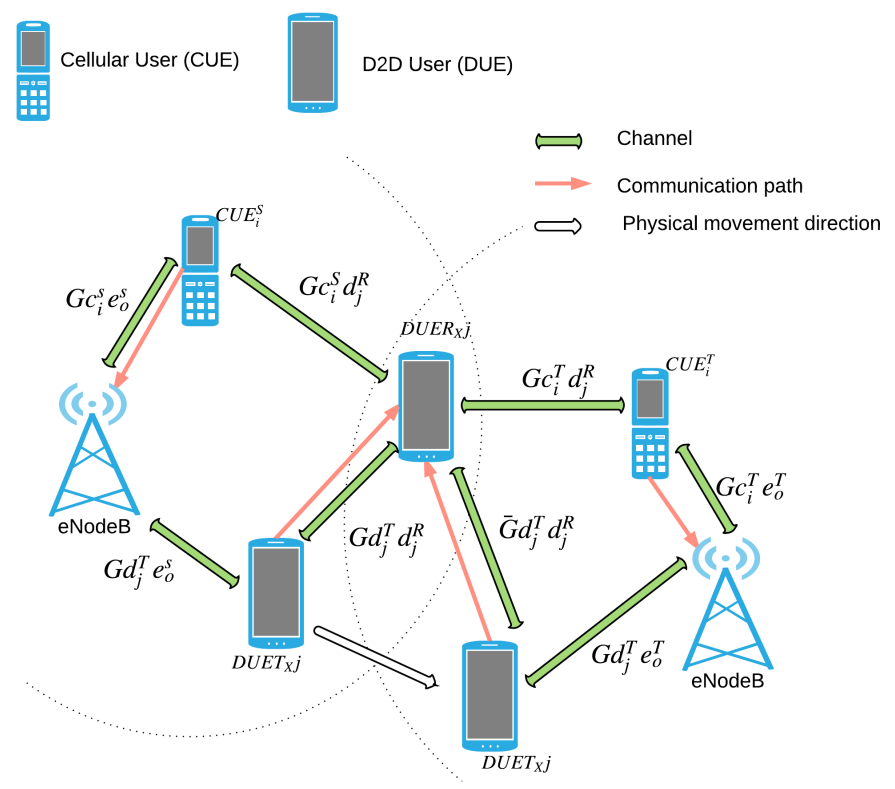

Fig. 3. Proposed mobility model.

Consider the total number of DUEs pair (each pair consists of one transmitter and one receiver) and CUEs are $N_{c}$ and $N_{d}$ respectively. Assuming a total number of resource sharing pair (one resource sharing pair consists of one D2D pair and one cellular user) is constant in the source cell as well as in target cell. That means once handover process is performed, a total number of devices in the target cell are as same as in the previous cell before the handover. In that case, we can apply all the operational mechanism at the target cell as in the source cell.

Assume that $f_{i j}$ is a specific resource sharing pair consists of $i$ th CUE and $j$ th DUE pair where $i \in 1, . . N_{c}$ and $j \in 1, . . N_{d}$. According to the Fig. 3, channel link indicates the specific gain between the particular devices or eNB. We consider $d_{j}^{T}$ is the D2D transmitter and $d_{j}^{R}$ is referred to as D2D receiver. We also consider $c_{i}^{S}$ and $c_{i}^{T}$ indicate cellular user of source cell and target cell respectively. $e_{o}^{S}$ and $e_{o}^{T}$ are the source and target cell respectively. Cellular user for the target cell is indicated as $C U E_{i}^{T}$ for the source it is $C U E_{i}^{S} . D U E T_{x j}$ is the $\mathrm{D} 2 \mathrm{D}$ transmitter, and $D U E R_{x j}$ is the receiver of the same cell. The channel gain between D2D users at the source cell is $G d_{j}^{T} d_{j}^{R}$ whereas after the handover takes place in the target cell it is $\bar{G} d_{j}^{T} d_{j}^{R}$. As the number of devices is not changing in the new cell, we assume $i$ and $j$ remain same after the handover takes place.

An adequate power transmit considering the pathloss and shadowing can be expressed as the following equation [15] [2].

$$
\mathbf{P}_{D}=\mathbf{S}_{D} \circ \overline{\mathbf{P}}_{\mathbf{D}}
$$


where, $\circ$ is a Hadamard operator and $\mathbf{S}_{D}$ is the result of resource scheduling for DUEs at the target cell. $\overline{\mathbf{P}}_{D}$ is a matrix constructed by eNB based on the all users within the target cell and it consists the values required power of each $f_{i j}$.

Since all the calculations related to the target cells are almost similar to the source cell during the normal operation of D2D mode, the only latency required is for taking the handover decision and relevant gain calculations which are as like as normal handover process. As both the cellular user and D2D users use same uplink resources, the spectrum efficiency would be the highest and sum-rate would also be maximum.

\section{Simulation PARAMETERS AND RESUltS}

In our previous paper [2], we proposed four possible moving directions, but we considered only one direction for the mathematical model as per Fig. 3. However, we considered all possible directions for our simulation. Based on the power allocation as stated in [2], we simulated the possible handover attempts done by the mobile station as only D2D aware handover solution was considered.

We also consider that none of the devices is out of the network area at the beginning and if a device moves towards out of the network coverage area but still in the proximity areas of another D2D pair, it may act as a relay.

Regarding the best mode selection, we proposed five possible operational conditions that could have occurred. These are Cellular mode, D2D mode, Relay mode, Waiting mode and Drop mode. The algorithm for the proposed simulation model to select the best operational mode are as follows.

Algorithm 1 shows the simplified way of how modes were selected. Selection of modes depends on mainly the threshold value that is set and the total path loss from the moving device to the base station. Fig. 5 shows the simulations results, and it indicates clearly the superiority of D2D mode which is the primary target of our model.

For the high Signal to Noise Ratio (SNR) and full load, we have the following equation [16].

$$
\operatorname{RSRP}(\mathrm{dBm})=\operatorname{RSSI}(\mathrm{dBm})-10 * \log (12 * \mathrm{~N}) .
$$

Here, RSRP is the Reference Signal Receive Power and RSSI indicates Received Signal Strength Indicator which can be again calculated by the below equation.

RSSI $=$ wideband power $=$ noise + serving cell power + interference power.

Reference Signal Received Quality (RSRQ) is determined by the following equation.
Data: Source eNodeB: Total loss between D2D pairs = Source $L_{D 2 D}$, Threshold between D2D pair = SourceTh $h_{D 2 D}$, Total loss between D2D transmitter and eNodeB $=$ Source $L_{D_{T} e N B}$, Threshold between D2D transmitter and eNodeB = SourceTh $h_{D_{T} e N B}$, Total loss between D2D receiver and eNodeB $=$ Source $L_{D_{R} e N B}$, Threshold between D2D receiver and eNodeB = SourceTh $h_{D_{R} \text { eNB }}$. Target eNodeB: Total loss between D2D pairs $=$ Target $L_{D 2 D}$, Threshold between D2D pair $=$ TargetT $h_{D 2 D}$, Total loss between $\mathrm{D} 2 \mathrm{D}$ transmitter and eNodeB =

Target $L_{D_{T} e N B}$, Threshold between D2D transmitter and eNodeB $=$ Target $T h_{D_{T} e N B}$, Total loss between $\mathrm{D} 2 \mathrm{D}$ receiver and eNode $\mathrm{B}=$ Target $L_{D_{R} e N B}$, Threshold between D2D receiver and eNodeB $=$ TargetTh $h_{D_{R} e N B}$,

if Source $L_{D 2 D} \leq$ SourceTh $h_{D 2 D}$ and Source $L_{D_{T} e N B}$ $\leq$ SourceTh $_{D_{T} e N B}$ and Source $L_{D_{R} e N B} \leq$

SourceTh $h_{D_{R} e N B}$ then

I Select D2D Mode;

else

| Search for the next possible mode

end

if Source $_{D 2 D}>$ SourceTh $h_{D 2 D}$ and Source $L_{D_{T} e N B}$ $\leq$ SourceTh $h_{D_{T} e N B}$ and Source $L_{D_{R} e N B} \leq$

SourceTh $h_{D_{R} e N B}$ then

| Select cellular Mode;

else

| Search for the next possible mode

end

if Source $_{D 2 D} \leq$ SourceTh $h_{D 2 D}$ and Source $L_{D_{T} e N B}$ $>$ SourceTh $_{D_{T} e N B}$ and Source $L_{D_{R} e N B} \leq$

SourceTh $h_{D_{R} e N B}$ then

Select waiting mode to observe the UE's moving direction;

else

| Search for the next possible mode

end

if Source $L_{D_{T} e N B}>$ SourceTh $h_{D_{T} e N B}$ and

Target $L_{D_{T} e N B} \leq$ TargetTh $h_{D_{T} e N B}$ and

Target $L_{D_{R} e N B} \leq$ Target $T h_{D_{R} e N B}$ then

| Prepare and trigger for the group handover;

else

| Search for the next possible mode

end

if Source $L_{D_{T} e N B}>$ SourceTh $h_{D_{T} e N B}$ and

Target $L_{D_{T} e N B}>$ Target $T h_{D_{T} e N B}$ and

Target $L_{D_{R} e N B}<$ TargetTh $h_{D_{R} e N B}$ and

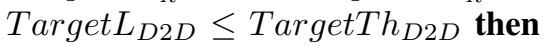

| Select Relay Mode;

else

I Call Drop;

end

Algorithm 1: Mode selection algorithm. 
RSRQ $=\mathrm{N} \times$ RSRP / RSSI, where, $\mathrm{N}$ mentions the number of physical resource block (PRBs).

Total path loss is calculated according to the paper [15].

$$
\text { Pathloss }(\mathrm{PL})=\alpha \cdot P L_{L O S}+(1-\alpha) \cdot P L_{N L O S},
$$

where, $\alpha$ is the average ratio of D2D users in line of sight (LOS) and non line of sight (NLOS) regions. $P L_{L O S}$ and $P L_{N L O S}$ are the path loss for the line of sight and non line of sight distance respectively and calculated by

$$
P L_{L O S / N L O S}=K_{1}+K_{2} \cdot \log _{10} \cdot d,
$$

where, $K_{1}$ and $K_{2}$ are the path loss coefficients and calculated from the receiving and transmitting antennas and frequency used. $d$ is the distance between the D2D users.

Total calculated shadowing loss is as follows:

$$
L(r)=R(\Delta r) \cdot L(r-\Delta r)+\sqrt{1-(R(\Delta r))^{2}} \cdot \sigma \cdot X
$$

where, $X$ is a Gaussian random variable with unit variance and zero-mean. $\sigma$ is standard deviation. The calculation of $R(\Delta r)$ is referred to the paper [15] which depends on the decorrelation distance of shadow fading and distance between the locations.

Multi-path fading is calculated by the equation as:

$$
M L=10 \log (-\log Y)
$$

where, $Y$ is a random variable with uniform distribution.

We have designed a MATLAB(R2016a) simulation model based on the proposed algorithm with the help of LTE System Toolbox. For some cases, we also used Simulink. Fig. 4 shows the MATLAB model where random moving directions are observed as an animation. In this figure, we considered a D2D pair and a cellular user. One of the D2D users who mainly transmits are moving randomly. For the simulation, we consider only one direction to avoid the complexity. We set two base stations within the area, and they are placed at different heights. The parameters used for the simulations are in Table I.

Fig. 6 shows the sum-rate trend while handover is taking place. It points that handover takes place without any major degradation of data throughput.

Fig. 7 also indicates the trend of path loss over distance. It shows that path loss is increasing when D2D transmitter is going apart from the source base station. The handover

\begin{tabular}{|c|c|}
\hline Simulation Parameters & Values \\
\hline Duplexing & TDD \\
\hline Walking model & Random \\
\hline Total loss & path loss + shadow fading + multi-path [15] \\
\hline Cellular User's location & {$\left[\begin{array}{llll}15 & 35 & 25\end{array}\right]$} \\
\hline D2D User's location & {$\left[\begin{array}{llll}40 & 50 & 60\end{array}\right]$} \\
\hline Source Base station's location & $(80,15,10)$ \\
\hline Target Base station's location & $(20,80,20)$ \\
\hline Carrier Frequency & $2 \mathrm{GHz}$ \\
\hline Handover Threshold & $114 \mathrm{dBm}$ \\
\hline Handover trigger & D2D aware \\
\hline Handover type & Group handover \\
\hline Resource used & Uplink \\
\hline Types of D2D & Underlay Inband \\
\hline Temperature & $290 \mathrm{~K}$ \\
\hline SINR & $-20 \mathrm{~dB}$ \\
\hline Maximum UE transmit power & $21 \mathrm{dBm}$ \\
\hline Transmit power or eNodeB & $39 \mathrm{dBm}$ \\
\hline Network Layout & 2 Sites and no grid maintained \\
\hline Total number CUEs per cell & 1 \\
\hline$K_{1}$ & $17.5 \mathrm{~dB}(\mathrm{NLOS})$ and $38.8 \mathrm{~dB}$ (LOS) \\
\hline$K_{2}$ & $43.3 \mathrm{~dB}(\mathrm{NLOS})$ and $16.9 \mathrm{~dB}$ (LOS) \\
\hline Total number DUEs pair per cell & 2 \\
\hline$\sigma$ & $3 \mathrm{~dB}$ (LOS and NLOS) \\
\hline MATLAB version & $2016 a$ \\
\hline Number of resource blocks & 100 resource block $/ 20 \mathrm{KHz}$ (Full) \\
\hline Total simulation performed & 500 \\
\hline
\end{tabular}
threshold point is also mentioned there.
TABLE I

SIMULATION PARAMETERS.

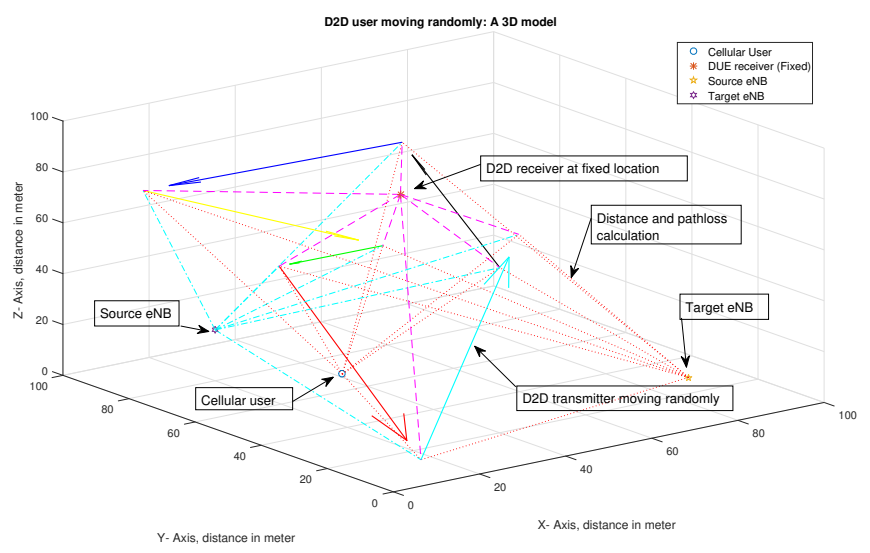

Fig. 4. MATLAB animation of proposed model

\section{CONCLUSiON}

In this paper, we started with reviewing the D2D communication system where mobility management is a big issue to be solved. Then, we studied the recent papers regarding the mobility management issues and highlighted our model previously published that might fit with the next generation cellular network standard. Then we put the simulation result with the animation model which is the primary focus of this paper. Simulation results show the different types of occurrences with the proposed model where D2D mode takes place for maximum times while the mobile station is moving. Our next work is to relate this proposed model to Markovian Chain model to validate the algorithm more extensively. 


\section{Percentage of different modes}

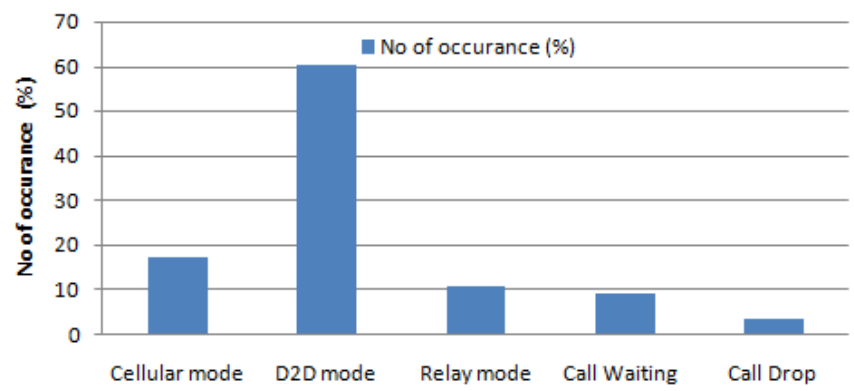

Fig. 5. Plot for the occurances (percentage)

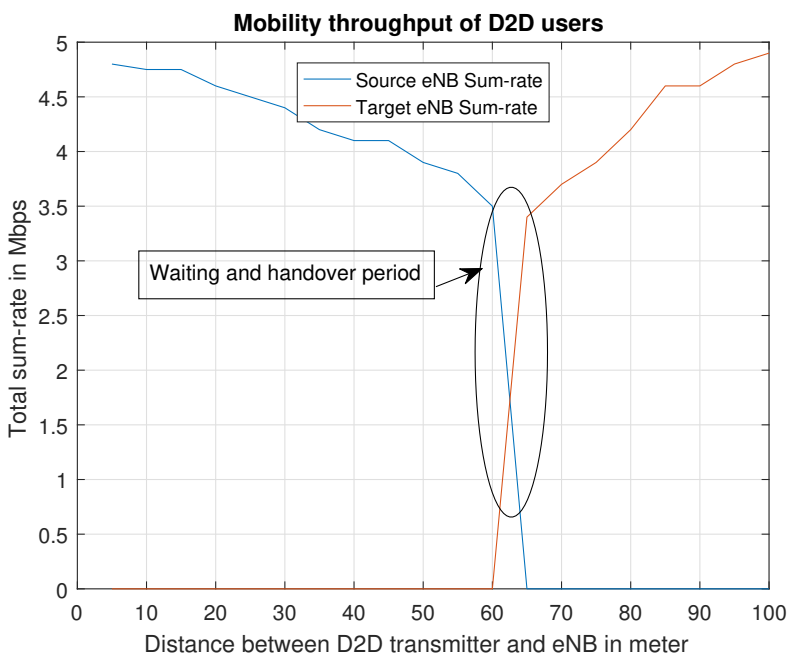

Fig. 6. Mobility throughput of D2D users

\section{REFERENCES}

[1] F. Boccardi, R. W. Heath, A. Lozano, T. L. Marzetta, and P. Popovski, "Five disruptive technology directions for 5g," Communications Magazine, IEEE, vol. 52, no. 2, pp. 74-80, 2014.

[2] S. Barua and R. Braun, "A novel approach of mobility management for the d2d communications in 5g mobile cellular network system," in 2016 18th Asia-Pacific Network Operations and Management Symposium (APNOMS), pp. 1-4, Oct 2016

[3] A. Asadi, Q. Wang, and V. Mancuso, "A survey on device-to-device communication in cellular networks," Communications Surveys \& Tutorials, IEEE, vol. 16, no. 4, pp. 1801-1819, 2014.

[4] S. Barua, S. C. Lam, P. Ghosa, S. Xing, and K. Sandrasegaran, "A survey of direction of arrival estimation techniques and implementation of channel estimation based on scme," in Electrical Engineering/Electronics, Computer, Telecommunications and Information Technology (ECTICON), 2015 12th International Conference on, pp. 1-5, June 2015.

[5] S. Barua and R. Braun, "Direction of arrival (doa) and channel estimation," in Self-Organized Mobile Communication Technologies and Techniques for Network Optimization, pp. 216-235, IGI Global, 2016.

[6] D. Wu, L. Zhou, Y. Cai, R. Hu, and Y. Qian, "The role of mobility for d2d communications in lte-advanced networks: Energy vs. bandwidth efficiency," Wireless Communications, IEEE, vol. 21, no. 2, pp. 66-71, 2014.

[7] J. Lim and D. Hong, "Mobility and handover management for heterogeneous networks in lte-advanced," Wireless personal communications, vol. 72, no. 4, pp. 2901-2912, 2013.

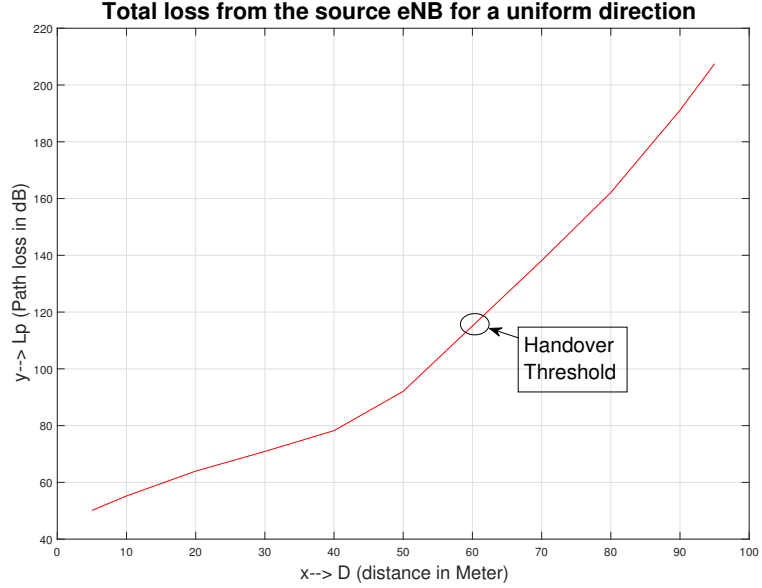

Fig. 7. Plot for the pathloss

[8] P. Ghosal, S. Barua, R. Subramanian, S. Xing, and K. Sandrasegaran, "A novel approach for mobility management in lte femtocells," International Journal of Wireless \& Mobile Networks, vol. 6, no. 5, p. 45, 2014.

[9] O. N. Yilmaz, Z. Li, K. Valkealahti, M. A. Uusitalo, M. Moisio, P. Lundén, and C. Wijting, "Smart mobility management for d2d communications in 5g networks," in Wireless Communications and Networking Conference Workshops (WCNCW), 2014 IEEE, pp. 219223, IEEE, 2014

[10] H. Y. Chen, M. J. Shih, and H. Y. Wei, "Handover mechanism for deviceto-device communication," in 2015 IEEE Conference on Standards for Communications and Networking (CSCN), pp. 72-77, Oct 2015.

[11] S. C. Lam, R. Subramanian, K. Sandrasegaran, P. Ghosal, and S. Barua, "Performance of well-known frequency reuse algorithms in lte downlink 3gpp lte systems," in Signal Processing and Communication Systems (ICSPCS), 2015 9th International Conference on, pp. 1-5, Dec 2015.

[12] A. U. Ahmed, M. T. Islam, M. Ismail, and M. Ghanbarisabagh, "Dynamic resource allocation in hybrid access femtocell network," The Scientific World Journal, vol. 2014, 2014.

[13] F. Afroz, S. Barua, and K. Sandrasegaran, "Performance analysis of fls, exp, log and m-lwdf packet scheduling algorithms in downlink 3gpp lte system," International Journal of Wireless \& Mobile Networks, vol. 6 , no. 5, p. 77, 2014.

[14] S. Barua, F. Afroz, S. S. Islam, A. U. Ahmed, P. Ghosal, and K. Sandrasegaran, "Comparative study on priority based qos aware mac protocols for wsn," International Journal of Wireless \& Mobile Networks, vol. 6, no. 5, p. 175, 2014.

[15] G. Jaheon, S. J. Bae, S. F. Hasan, and M. Y. Chung, "A combined power control and resource allocation scheme for $\mathrm{d} 2 \mathrm{~d}$ communication underlaying an lte-advanced system," IEICE Transactions on Communications, vol. 96, no. 10, pp. 2683-2692, 2013.

[16] "http://www.laroccasolutions.com/78-rsrp-and-rsrq-measurement-inlte/,’ 20.05.2017. 